\title{
Study on Key Image Information Positioning Method based on Template Matching and Gabor Filtering
}

\author{
Zhao Lei ${ }^{1, a}$ and Ren Hong-e $e^{1 *}$ \\ ${ }^{1}$ Northeast Forestry University, Harbin, China \\ 154372732@qq.com,renhonge163@163.com
}

\begin{abstract}
Eye location gradually becomes a hot research topic of image analysis field. Images gathered in face recognition have low recognition rate due to multi-pose, uneven illumination and ornament. Firstly, eye coarse location based on eye template is introduced. Then, Gabor filter is utilized to carry out pupil location of coarsely located eye. The experimental results show the combination of two methods improves location accuracy of eyes and owns good adaptation and stability.
\end{abstract}

Keywords: template; eye location; Gabor filtering; pupil

\section{Introduction}

With technological development, eye location and recognition technology gradually becomes a hot research topic of image processing and video analysis field. The eye as an important face feature organ of human can be utilized to infer and reflect various kinds of mental activity and spiritual state information from its tiny changes. In present key techniques for monitoring drivers' state of fatigue, detection of drivers' state of fatigue through analyzing drivers' eye state is a research hotspot of fatigue state detection field. Drivers will generate fatigue after long-term continuous driving. Fatigue is a kind of normal physiological activity law and stress response of self-protection caused by excessive manual labor or brain work. At this time, eye state such as the increase in the time for closing eyes, the decrease in blinking frequency and low concentrate can be used to speculate drivers' degree of fatigue. Thus, this topic aims to conduct real-time monitoring of drivers' eye state through eye location and tracking so as to prevent and completely eradicate fatigue driving. Hence, accuracy and robustness of drivers' eye location will directly influence usability of fatigue detection system.

\section{Coarse Location based on Eye Template}

Face distribution is a regular geometrical shape. The eyebrow, eyes, nose and mouth are distributed at the vertical direction. Usually, distribution position of these organs is fixed. During locating eyes, we have the following skills to analyze face image:

(1) The eyes are at the upper half of face;

(2) The left and right eyes are horizontally and symmetrically distributed at both sides of face region. The angle between the center line of two eyes and horizontal plane will not be too large;

(3) Height-width ratio of eyes has a certain range;

(4) Eye width is roughly between $1 / 4$ and $1 / 5$ of face width.

(5) For eye grayscale projection, black pixels in the eye region account for over $40 \%$ of

* Corresponding Author 
all pixels.

Firstly, initial position of eyes should be gained. Based on detection of face, search matching is carried out on the upper half of face region according to existing priori knowledge about face and eye location skills. The initial position of eyes can be detected through judging whether positional relationship between two eyes meets the conditions. Meanwhile, coordinate, width and height at upper left corner of two rectangle boxes are recorded to lay a foundation for follow-up researches[1,5].

Therefore, the primary task of template matching is to collect eye template images. Then, it is necessary to segment sub-images whose size is the same with template images from each position of face image inputted, calculate correlation coefficient between sub-images and eye template and take the position with the largest correlation coefficient as eye position. But such simple template matching method has poor stability and is very sensitive to changes in eye expression, rotation and light. To solve these problems, R.Brunelli et. al., introduced the concept of multi-scale template in their researches and partly solved scale change problem through scaling original template image to 5 different sizes in proportion. Deformable template method is applicable to extraction of face components. Simulated diagram of eye template is established through analysis of eye shape, as shown in Figure 1[2,6].

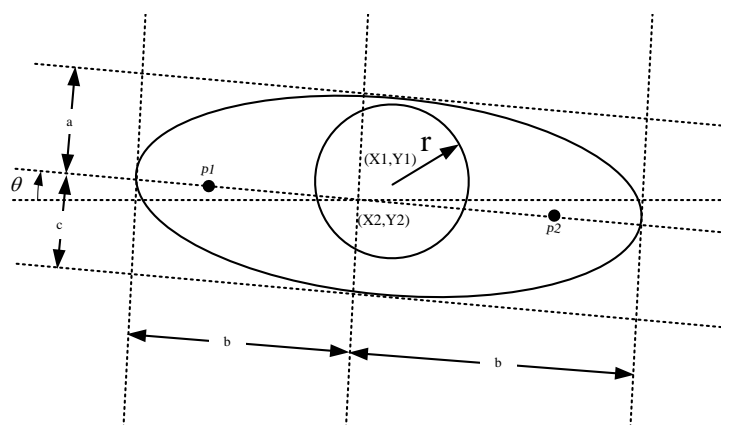

Figure 1. Eye Template

The margin is two parabolas, and the circle in the middle of eye is pupil. $(X 1, Y 1)$ means the center of pupil circle, and $\mathrm{r}$ means radius of pupil circle. For the upper and lower parabolas, $((X 2, Y 2), a, b, c, \theta) .(X 2, Y 2)$ means center coordinate of eye template. a means the largest height of upper parabola; $b$ means a half of template length; $\mathrm{c}$ means the highest height of lower parabola; $\theta$ means the included angle between the template and horizontal direction. The template interacts with input image, its trough, crest and margin area. The trough, crest and margin correspond to black area, white area and eye contour of eyes. Energy function established according to this template needs to take into account of eye features in actual image and prior relationship among each parameter.

In this method, values of the above eye parameters are corrected through dynamic interactive adaptation of grayscale distribution features of template and image, margin, trough and crest. Energy function is first established in line with priori knowledge of eyes. It can reflect fitting degree of eye template and eye image. Values of these parameters are confirmed through energy function minimization $[3,7,8]$.

Energy function $E_{c}$ is defined as below:

$$
E_{c}=E_{v}+E_{e}+E_{i}+E_{p}+E_{q}
$$


Where, $E_{v}$ reflects the energy of trough in the image. It is defined as follows:

$E_{v}=-\frac{C_{1} \int_{R_{c}} \Phi_{v}(\vec{x}) d A}{\left|R_{c}\right|}$

(2)

$E_{e}$ reflects the energy of margin in the image. It is defined as follows:

$E_{e}=-\left(\frac{c_{2} \int_{\partial R_{b}} \Phi_{e}(\vec{x}) d s}{\partial R_{b}}+\frac{c_{3} \int_{\partial R_{w}} \Phi_{e}(\vec{x}) d s}{\left|\partial R_{w}\right|}\right)$

$E_{t}$ reflects the energy of original grayscale image. It is defined as follows:

$E=\frac{C_{4} \int_{R_{b}} \Phi_{t}(\vec{x}) d A}{\left|R_{b}\right|}-\frac{C_{5} \int_{R_{w}} \Phi_{t}(\vec{x}) d A}{\left|R_{w}\right|}$

(4)

$E_{p}$ reflects the energy of crest in the image. It is defined as follows:

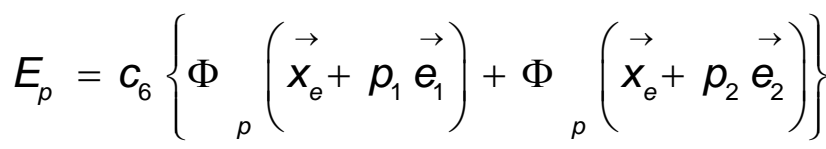

(5)

$E_{q}$ reflects the prior energy, or called internal energy. It is defined as follows:

$$
\begin{aligned}
E_{q}= & k_{1}\left\|\vec{x}_{e}-\vec{x}_{c}\right\|^{2}+k_{2}\left[p_{1}-p_{2}-(r+b)\right]^{2}+k_{3}(b-2 r)^{2} / 2 \\
& +k_{4}\left[(b-2 a)^{2}+(a-2 c)^{2}\right]
\end{aligned}
$$

(6)

In formulas (2) - (6), $\Phi_{t} \cdot \Phi_{v} \cdot \Phi_{e}$ and $\Phi_{p}$ represent luminance image as well as trough image, margin image and crest image generated through morphological operation, respectively; $R_{b}, R_{w}, \partial R_{b}$ and $\partial R_{w}$ represent iris area, eye white area, iris boundary and eye white boundary, respectively. Their area or length is expressed with $\left|R_{b}\right|,\left|R_{w}\right|$, $\left|\partial R_{b}\right|$ and $\left|\partial R_{w}\right|$, respectively. A and s represent area and arc length. $\overrightarrow{e_{1}}$ and $\overrightarrow{e_{2}}$ are unit vector of two directions, i.e.

$$
\overrightarrow{e_{1}}=(\cos \theta, \sin \theta) \quad, \quad \overrightarrow{e_{2}}=(-\sin \theta, \cos \theta)
$$

Deformable template can not only achieve eye location, but also can gain eye size and 
shape information. When the minimum energy function appears, eye features are extracted through calculating the number of points in parameter control which consist of template parameters. But this method just coarsely locates eyes, and fails to solve the problem of initial parameter confirmation for the template. Moreover, convergence of optimization process of energy function depends on selection of initial position of template to a large extent. Hence, after coarse location of eyes, this paper introduces Gabor filtering to further process ye image. Eye coarse location results are shown Figure 2, Figure 3, Figure 4 and Figure 5.
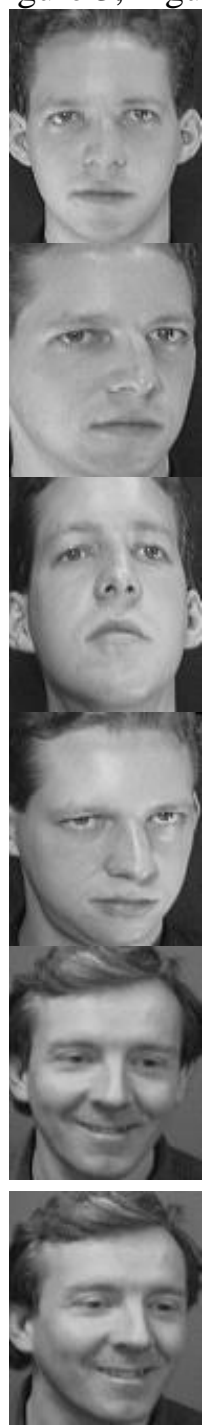
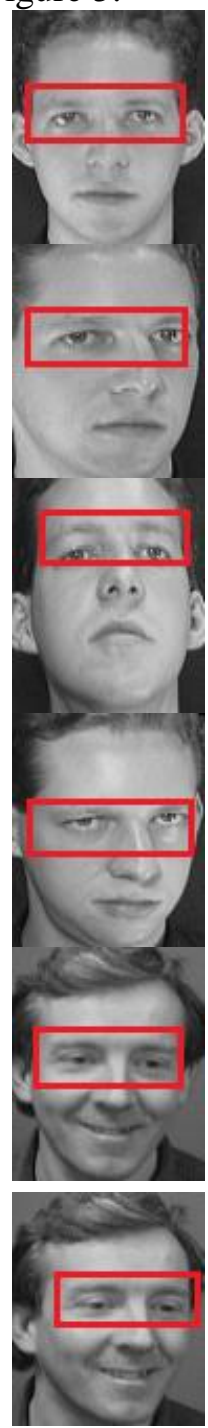
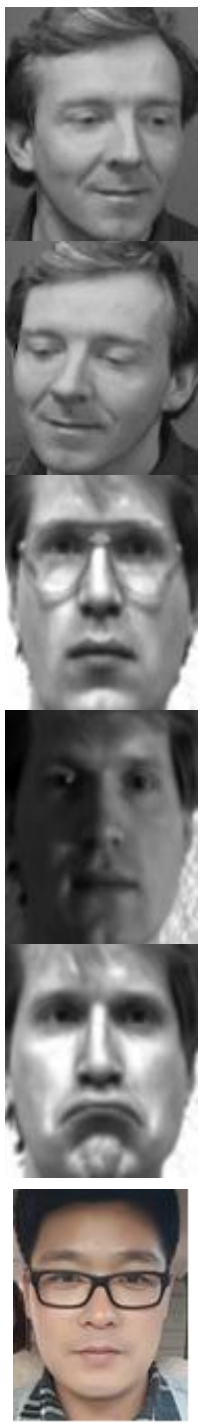

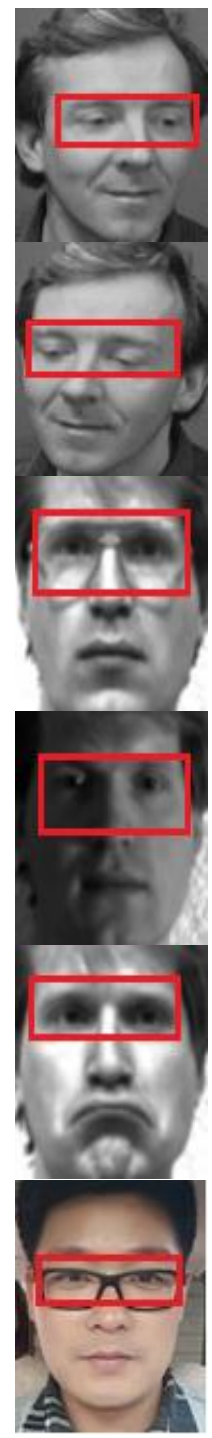

Figure 2. Eye Detection Results under Multi-Pose, Multi-Expression and Ornaments 

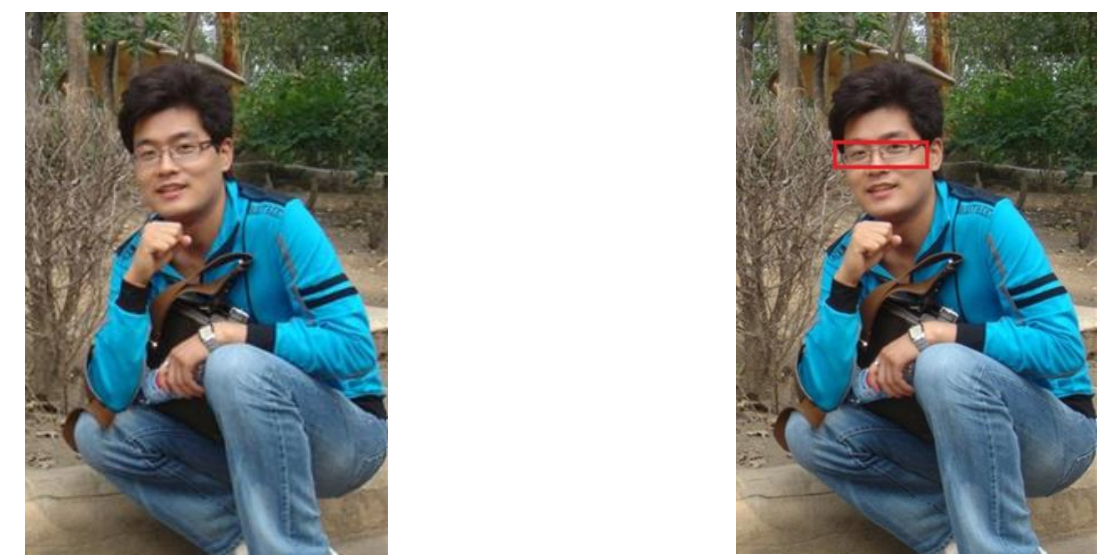

Figure 3. Detection Result under Complicated Background

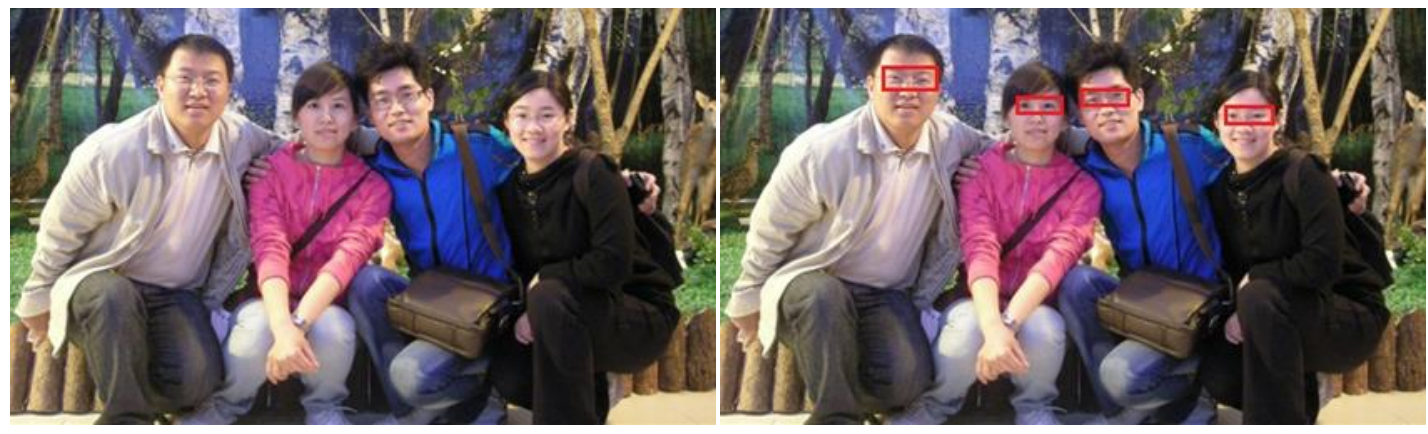

Figure 4. Multi-Eye Detection Result of under Complicated Background
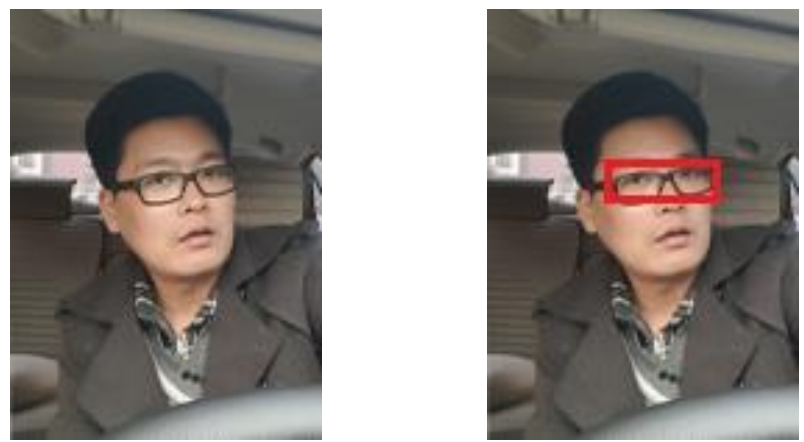

Figure 5. Eye Detection Result under Driving Environment

\section{Pupil Location based on Gabor Filtering}

\subsection{Gabor Filter Bank}

Odd symmetrical Gabor filters are adopted to extract textural features of images:

$\psi\left(x, y, \sigma_{x}, \sigma_{y}, \omega\right)=\left(1 / 2 \pi \sigma_{x} \sigma_{y}\right) \exp \left[-\frac{1}{2}\left(\frac{\sigma_{y}^{2} x^{2}+\sigma_{x}^{2} y^{2}}{\sigma_{x}^{2} \sigma_{y}^{2}}\right)\right] \sin (\omega x)$

Where, $\omega$ is modulation frequency of Gaussian function (or called center frequency of filter); $\sigma_{x}$ and $\sigma_{y}$ are standard variances of Gaussian function along x direction and y direction, respectively which decide the size of Gaussian window. So they are also 
called scale factor. A group of self-similar filters can be gained through scale expansion and rotation transformation of Gabor primary function:

Scale expansion: $\omega=\omega_{\max } / f^{s}$

Rotation transformation: $x=x_{\theta} \cos \theta+y_{\theta} \sin \theta ; y=-x_{\theta} \sin \theta+y_{\theta} \cos \theta$. $\theta=r \pi / \mathrm{k} . \mathrm{k}$ and s represent direction number and scale number of filter bank. $r \in[0, k-1], f^{s}$ means bandwidth of filter presents exponential increase with the scale.

To reduce computation complexity, Gabor filter is decomposed to simple one-dimensional filter combination, and one-dimensional filtering is achieved through recursive filtering method with small calculation amount $[4,9,10]$. The expression of Gabor filter is directly written to the form of separated $\mu$ and $v$ variables.

$h(x, y)=q(\mu) * g\left(v, \sigma_{v}\right)$

(9)

Where, $q(\mu)$ and $g\left(v, \sigma_{v}\right)$ are:

$q(\mu)=\frac{1}{\sigma_{\mu} \sqrt{2 \pi}} \exp \left\{-\frac{\mu^{2}}{2 \sigma_{\mu}^{2}}\right\} \cos (\omega \mu)$

$g\left(v, \sigma_{v}\right)=\frac{1}{\sigma_{v} \sqrt{2 \pi}} \exp \left\{-\frac{v^{2}}{2 \sigma_{v}^{2}}\right\}$

When $\sigma_{\mu}=\sigma_{v}=\sigma, \mu^{2} / \sigma_{\mu}^{2}+v^{2} / \sigma_{v}^{2}=\left(x^{2}+y^{2}\right) / \sigma^{2}$. The following decomposed formula is gained through spreading cos term:

$h(x, y)=f_{1}(x) * f_{2}(y)-f_{3}(x) * f_{4}(y)$

Where, $f_{1}, f_{2}, f_{3}$ and $f_{4}$ are:

$f_{1}(x)=g(x, \sigma) \cos ((\omega \cos \theta) x)$

$f_{2}(y)=g(y ; \sigma) \cos ((\omega \sin \theta) y)$

$f_{3}(x)=g(x ; \sigma) \sin ((\omega \cos \theta) x)$

$f_{4}(y)=g(y, \sigma) \sin ((\omega \sin \theta) y)$

\subsection{Each Order Moment of Filter Coefficient}

For a sample $X$ of the random process, if $\mu_{k}=E\left[(X-E(X))^{k}\right]$ exists, it is $\mathrm{k}$-order central moment of $\mathrm{X}$. Each order moment of random process can measure general features: 
Traditional image retrieval technique based on Gabor filtering mostly extracts mathematical expectation $\mu_{s, \theta}$ of Gabor wavelet coefficient and secondary-order moment-variance $\sigma_{s, \theta}$ as textural features of images, i.e.

$\mu_{s, \theta}=\sum_{y=1}^{M} \sum_{x=1}^{N} g(x, y)_{s, \theta} / M N$

$\sigma_{s, \theta}=\sqrt{\sum_{y=1}^{M} \sum_{x=1}^{N}\left(g(x, y)_{s, \theta}-\mu_{s, \theta}\right)^{2} / M N}$

Here, third-order moment, fourth-order moment and energy of Gabor filter coefficient are introduced to define skewness $\delta_{s, \theta}$, kurtosis $_{s, \theta}$ and energy $e_{s, \theta}$ at each scale s and direction $\theta$, as follows:

$\delta_{s, \theta}=\mu_{3} / \sigma_{s, \theta}^{3}=\sum_{y=1}^{M} \sum_{x=1}^{N}\left(g_{s, \theta}(x, y)-\mu_{s, \theta}\right)^{3} / M N \mu_{s, \theta}^{3}$

$k_{s, \theta}=\mu_{4} / \sigma_{s, \theta}^{4}-3=\sum_{y=1}^{M} \sum_{x=1}^{N}\left(g(x, y)_{s, \theta}-\mu_{s, \theta}\right)^{4} / M N \sigma_{s, \theta}^{4}-3$

$e_{s, \theta}=\sum_{y=1}^{M} \sum_{x=1}^{N}\left(g(x, y)_{s, \theta}\right)^{2}$

Skewness, kurtosis and energy distribution measure texture distribution symmetry, steep degree and total energy at re-filtering scale and direction. Figure 6, Figure 7 and Figure 8 show frequency space distribution of Gabor filtering, frequency component, real component and imaginary component.

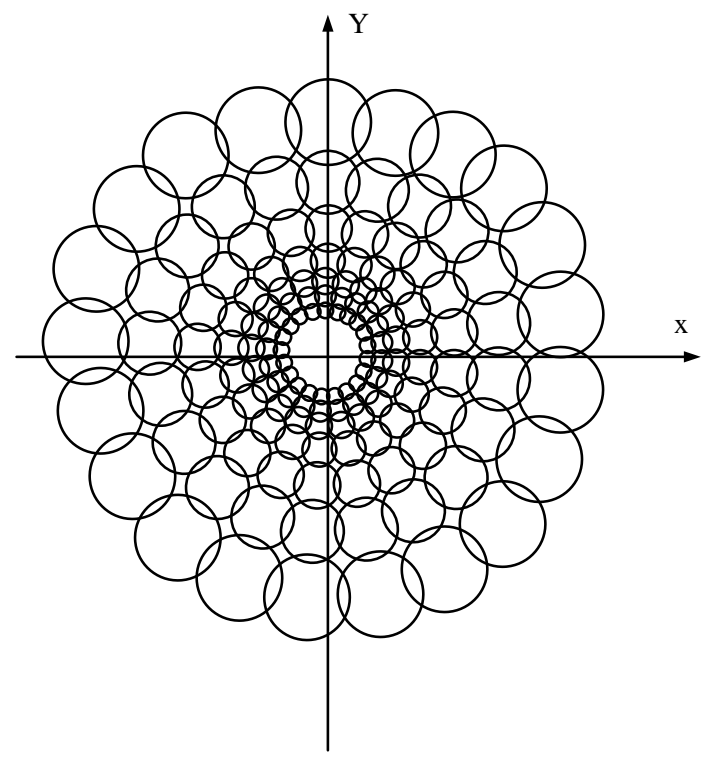

Figure 6. Frequency Space Distribution of Gabor Filtering 


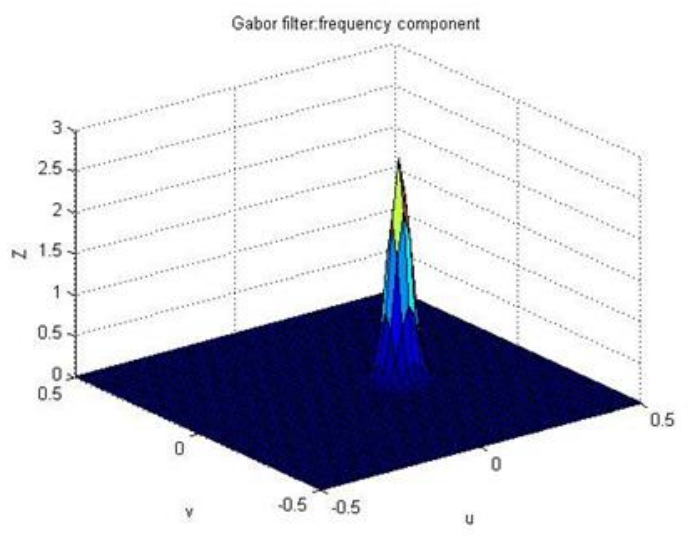

图 7 frequency component

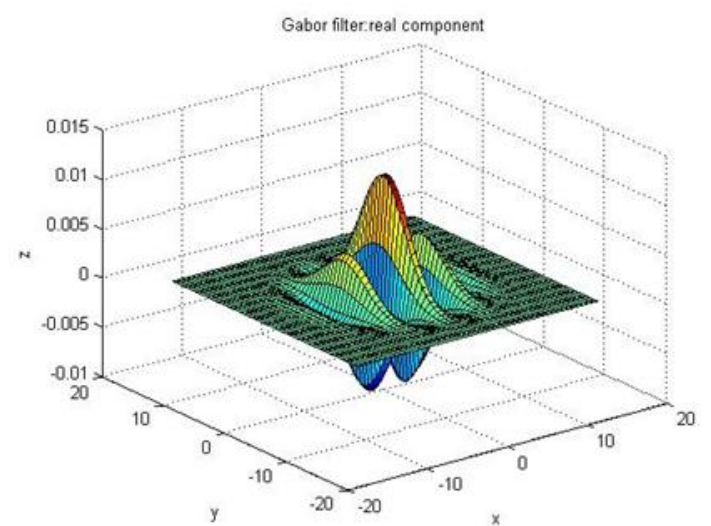

(a) real component

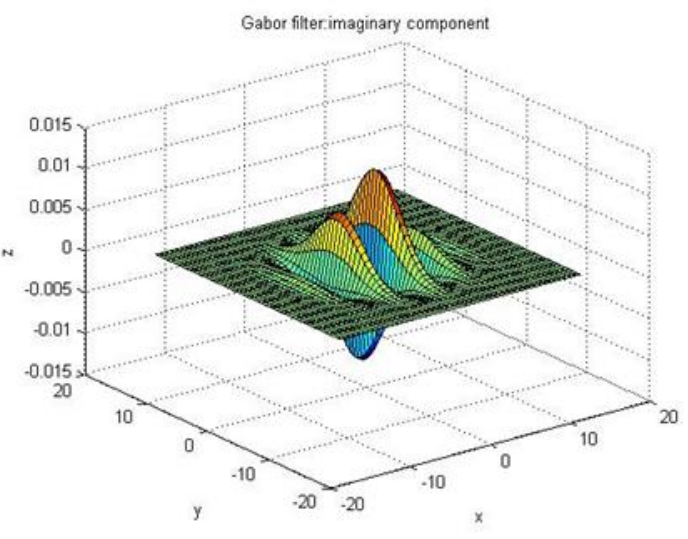

(b)imaginary component

\section{Figure 8. Real Component and Imaginary Component of Gabor Filtering}

\section{Experimental Results and Analysis}

It is very hard to choose a proper eye location database. This not just depends on the emphasis of research problem, but also relies on the database, because it influences whether experimental results are universal. This experiment adopts AT\&T's face database and Yale's face database as well as the photos shot by the author under driving environment as the objects. All images in the database are classified into post change, expression change, ornament change, illumination change and background change etc. It is known from analysis of experimental results that, first eye coarse location based on template and then accurate eye location by Gabor filtering can not just locate eyes accurately, but the processing speed boosts obviously. According to the analysis results of eye detection of 1000 faces respectively under multi-pose, illumination, glasses wearing (excluding sunglasses), under normal circumstances, eye location success probability under multi-pose is high, while illumination and ornament wearing impose great influence on eye location, as shown in Table 1, Table 2 and Table 3. Experimental results of accurate pupil location are shown in Figure 9, Figure 10, Figure 11 and Figure 12.

Table 1. Multi-Pose Detection Result

\begin{tabular}{|c|c|c|c|}
\hline Multi-pose & Accurate location & Wrong location & No location \\
\hline Quantity & 965 & 30 & 5 \\
\hline Percentage & $96.5 \%$ & $3 \%$ & $0.5 \%$ \\
\hline
\end{tabular}


Table 2. Illumination Detection Result

\begin{tabular}{|c|c|c|c|}
\hline Multi-pose & $\begin{array}{c}\text { Accurate } \\
\text { location }\end{array}$ & $\begin{array}{c}\text { Wrong } \\
\text { location }\end{array}$ & No location \\
\hline Quantity & 863 & 57 & 60 \\
\hline Percentage & $86.3 \%$ & $5.7 \%$ & $6 \%$ \\
\hline
\end{tabular}

Table 3. Ornament Wearing Detection Result

\begin{tabular}{|c|c|c|c|}
\hline Multi-pose & $\begin{array}{c}\text { Accurate } \\
\text { location }\end{array}$ & $\begin{array}{c}\text { Wrong } \\
\text { location }\end{array}$ & No location \\
\hline Quantity & 712 & 206 & 82 \\
\hline Percentage & $71.2 \%$ & $20.6 \%$ & $8.2 \%$ \\
\hline
\end{tabular}
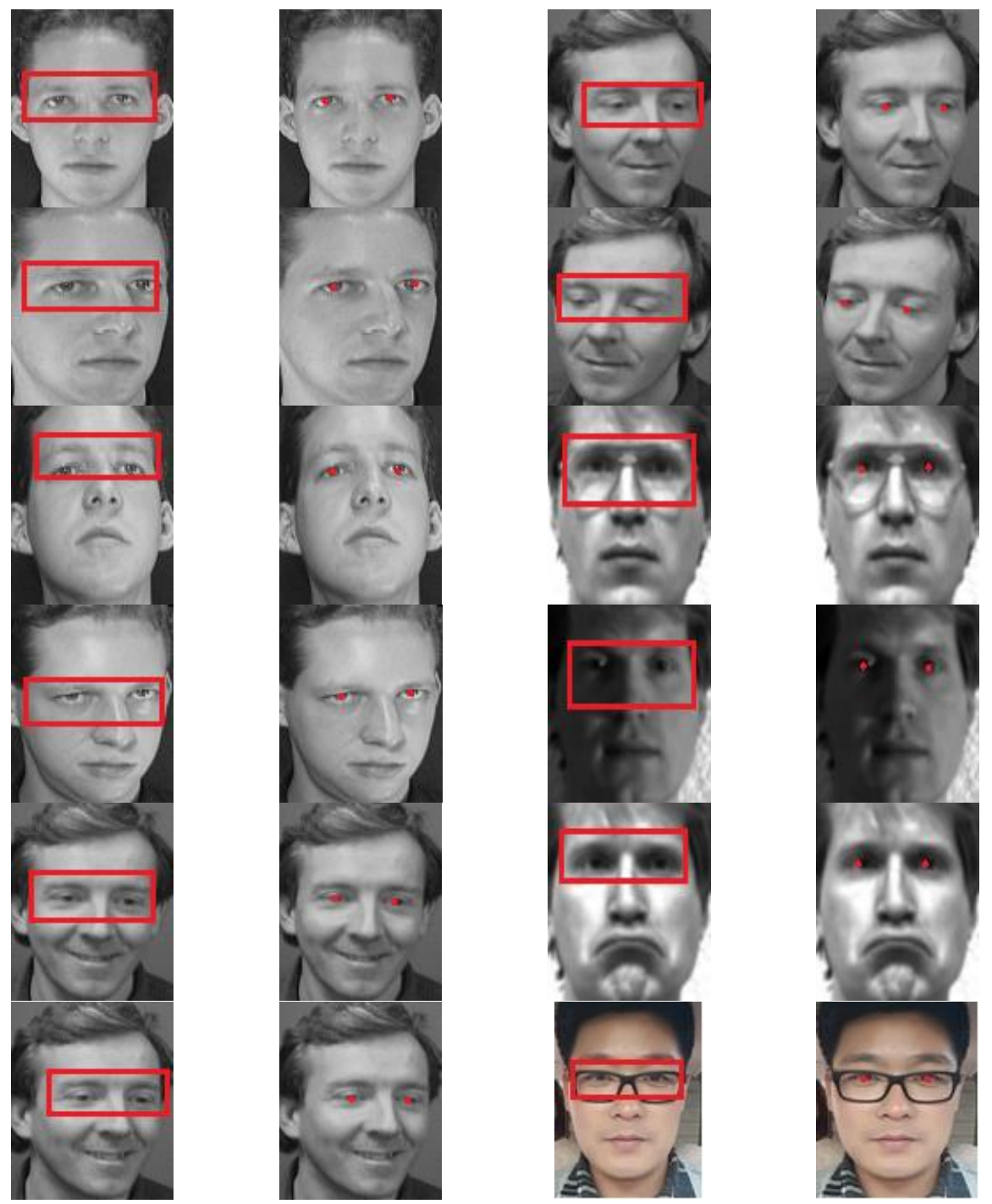

Figure 9. Detection Results under Multi-Pose, Multi-Expression and Ornaments 

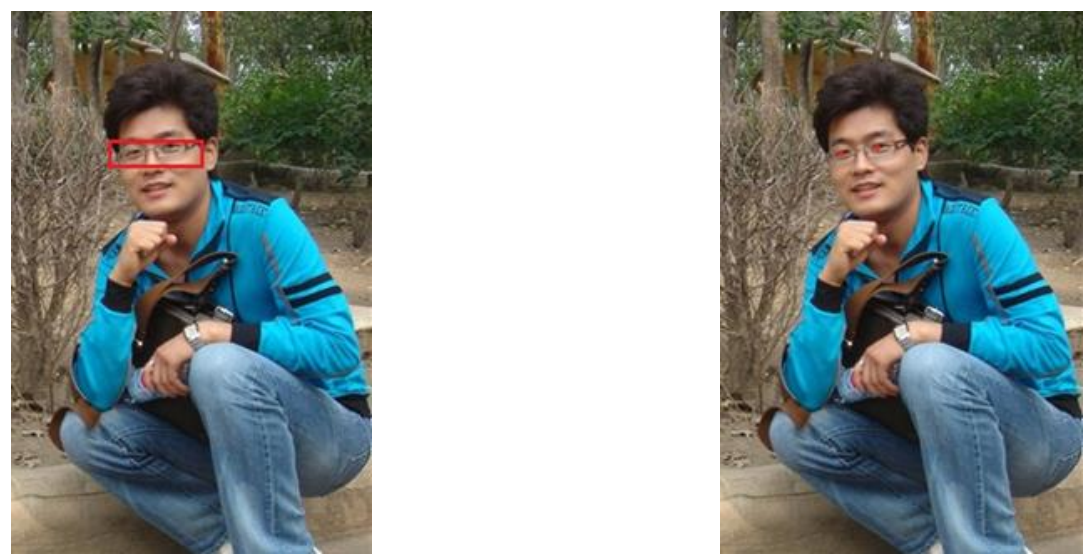

Figure 10. Detection Result under Complicated Background

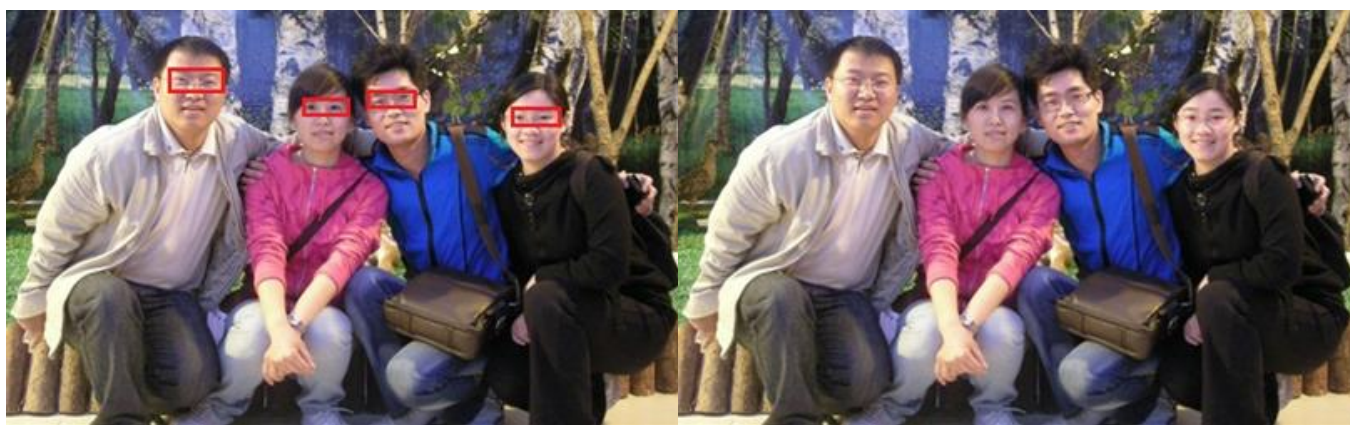

Figure 11. Multi-Eye Detection Result under Complicated Background
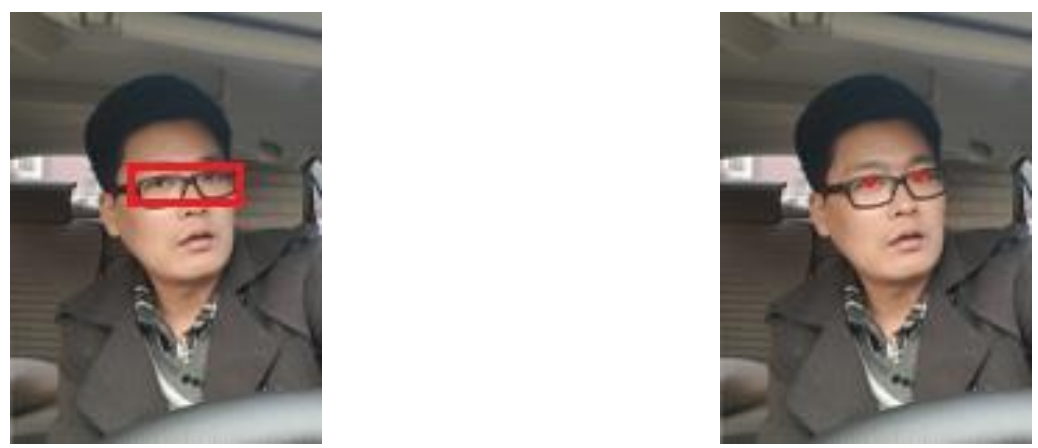

Figure 12. Eye Detection Result under Driving Environment

\section{Acknowledgement}

This work was supported by the key projects of Heilongjiang natural science foundation (ZD201203/C1603)

\section{References}

[1] L. Liling, "The Fatigue driving detection method based on driver's eye detetion", (2012), Electronical University.Sichuan.

[2] YU Tian tian, TANG Pu ying, "An eye orientation approach based on deformable template and genetic algorithms".Computer Simulation,vol.24,no.04,( 2007),pp.200-239.

[3] Song Jiatao, "Human eye localization and face recognition based on binary edge map",(2004), Zhe Jiang University,Zhejiang. 
[4] C. Xiao-Guang, "Feng Ju-Fu. Fast gabor filtering", Acta Automatica Sinica, vol.33, no.5, (2007), pp.456-461.

[5] Z. Yan, Chen Bo-na, "Image Retrieval Based on the High-order Moments of Gabor Filtering Coefficients", Opto-Electronic Engineering, vol.37, no.3, (2010), pp.79-87.

[6] Z.Yin-di, Zhang Liang-pei, Li Ping-xiang, "A Texture segmentation Algorithm Based on Directional Gabor Filters",Journal of Image and Graphics. 2006, vol.11 ,no.4,(2006),pp.504-510

[7] X. Wanyin, "A local characteristic scale selection method based on Gabor wavelets", Journal of image and graphics,vol.16,no.1, (2011), pp.72-78.

[8] H. Zengxi, "Method for fast automatic eyes localization", Computer Engineering and

[9] Applications, vol.47, no.7, (2011), pp.183-186.

[10] Y. Fan, "Eye location based on adaptive image segmentation and curve blending", Optics and Precision Engineering, vol.21,no.12, (2013), pp.3255-3262.

[11] T. Taizhe, "Human eyes localization based on morphological quotient template",Computer Application and Software, vol.32,no.1,(2015),pp.194-198.

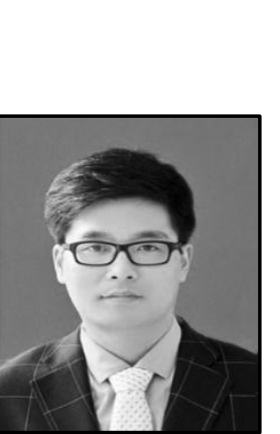

\begin{abstract}
Authors
Zhao Lei, He received his BS in Computer science and technology from Northeast Forestry University, Harbin, China, in 2005. He got his MS in Computer application technology from Northeast Forestry University, Harbin, China, in 2009. He got his ph.d in Forestry Engineering Automation from Northeast Forestry University, Harbin, China, in 2013.He is an Associate Professor in the School of Hei LongJiang International University. His research interests include pattern recognition and intelligent control.

Honge REN, He received the Ph.D. degree from Northeast Forestry University, China, in 2009. She is currently professor of information and computer engineering college at school of Northeast Forestry University, supervisor of Dr. Her main research interests include the pattern recognition and intelligent control.
\end{abstract}

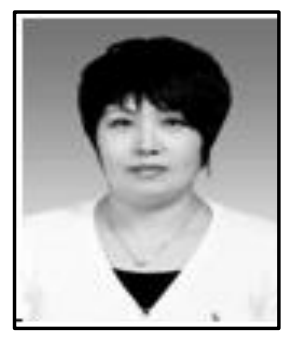


International Journal of Signal Processing, Image Processing and Pattern Recognition Vol.9, No.3 (2016) 